\title{
The best suggestion for COVID-19 in a non-infected pregnant population is still prevention: a commentary
}

\author{
Rui-Hong Xue ${ }^{1}$ and Hefeng Huang ${ }^{2}$ \\ ${ }^{1}$ International Peace Maternity and Child Health Hospital, School of Medicine, Shanghai \\ Jiao Tong University, Shanghai, China \\ ${ }^{2}$ International Peace Maternity and Child Health Hospital, School of Medicine,
}

October 9, 2020

The best suggestion for COVID-19 in a non-infected pregnant population is still prevention: a commentary

Rui-Hong Xue ${ }^{1}$, He-Feng Huang ${ }^{2,3,4,5}$

${ }^{1}$ Department of Obstetrics and Gynecology, International Peace Maternity and Child

Health Hospital, School of Medicine, Shanghai Jiao Tong University, Shanghai, China

${ }^{2}$ Center of Reproductive Medicine, International Peace Maternity and Child Health Hospital, School of Medicine, Shanghai Jiao Tong University, Shanghai, China

${ }^{3}$ Institute of Embryo-Fetal Original Adult Disease Affiliated to Shanghai Jiao Tong University School of Medicine, Shanghai Jiao Tong University, Shanghai, China

${ }^{4}$ Shanghai Key Laboratory of Embryo Original Diseases, Shanghai, China

${ }^{5}$ Shanghai Municipal Key Clinical Specialty, Shanghai, China

Correspondence: He-feng Huang, E-mail:huanghefg@hotmail.com;Postal address: No.910 Hengshan Road, Shanghai, China. Postal code: 200030. Work phone: +86-021-64070434

The author report no conflict of interest.

Word Count:395

Funding: None

COVID-19 is still spreading rapidly around the world, the global cases have been still increasing with millions of people losing lives. Pregnant women are a vulnerable group of people during the global pandemic with potential risks for both mothers and neonates. Though the management guidelines during pregnancy are evolving continuously, pregnant women suffered from COVID-19 mean worse pregnancy outcomes, both physically and mentally, especially during the first or second trimester, the patient or doctor may stick in the middle when facing with choices. We would like to further emphasize the importance of prevention.

By analyses of neonatal outcome in 29 pregnant women with COVID-19, 12 of 13 hospitalized neonates presented with radiological features for pneumonia through X-ray or CT screening, which suggested that intrauterine or intrapartum transmission is possible. Although a systematic review concluded that the rate of infection is no greater when the baby is born vaginally, breastfed or remains with the mother, the safety of mode of delivery among COVID-19 infected pregnant women remains controversial and calls for prospective study. 
Multiple COVID-19 vaccines are currently in phase 3 trials with efficacy assessed as prevention of virologically confirmed disease. Vaccines have been considered the promising way to effectively control the virus, however, the effectiveness and safety of vaccines or drugs to be used in pregnant women have been reported very few and need further investigations. As reported in an example that respiratory syncytial virus vaccination in pregnant women did not meet the prespecified success criterion for efficacy against RSV-associated, medically significant lower respiratory tract infection in infants up to 90 days of life. The suggestion of a possible benefit with respect to other end-point events involving RSV-associated respiratory disease in infants warrants further study. As confirmed that public health interventions could improve the control of the COVID-19 outbreak. Thus, the best suggestion in pregnancy in a non-effected population might be still physical distancing, face masks, and eye protection.

COVID-19 characters as strong infectivity, rapid and wide spread, and have caused significant morbidity and mortality. In the 1902 paper, Ballantyne said, "as with the premature, the ideal plan of procedure is prevention", so with the COVID-19. Before the effective and safety guard of vaccines of COVID-19 to be used in pregnant women, strong awareness of protection and effective measures to be taken among the non-infected population, the increasing trend might see the turning point.

\section{Contributions}

RH-X wrote the manuscript. HF-H was responsible for the design and supervision of the work and have made critical revision.

\section{Sources of funding}

None

\section{Disclosure of interests}

The author of this work declare no potential conflicts of interest relevant to this article.

\section{Ethics approval}

The manuscript was approved by the ethics committee on human research. Review Board: (GKLW) 2020-05 On March 15, 2020. 